\title{
Biological Characteristics of Jade Perch (Scortum Barcoo)
}

\author{
Jie Hu${ }^{1}$, Ningning Yan $^{1,2}$, Chengfei Sun ${ }^{1,2}$, Junjian Dong ${ }^{1}$ and Xing $\mathrm{Ye}^{1 *}$ \\ ${ }^{1}$ Key Laboratory of Tropical and Subtropical Fisheries Resource Application and Cultivation, Chinese Academy of Fishery China \\ ${ }^{2}$ College of Fisheries and Life Science, Shanghai Ocean University, China
}

Submission: October15, 2018; Published: October 26, 2018

Corresponding author: Xing Ye, Pearl River Fisheries Research Institute, Chinese Academy of Fishery Sciences, No. 1 Xingyu Road, Guangzhou 510385, China; Tel: +86-2081617091/13622802086; Fax: +86-20-81616162; Email: gzyexing@163.com

Abstract

Jade perch (Scortum Barcoo) is a highly nutritious fresh water bass species native in Australia and now being a promising candidate for aquaculture in China. To provide a fundamental biological basis for future germplasm improvement and exploitation of jade perch, biological characteristics of jade perch, including propagation, morphological and genetic characters were analyzed in this study. Jade perch becomes sexually mature at four-year-old. Zygotes of jade perch are transparent, buoyant, and swell upon absorption of water to up to $2.1 \pm 0.3 \mathrm{~mm}$ in diameter. At a water temperature of $27.5 \pm 2^{\circ} \mathrm{C}$, zygoteshatched $21-26 \mathrm{~h}$ after fertilization. Comparison of morphological characters between twoand six-month-old jade perch revealed that the proportion of the head with respect to the body decreased significantly $(\mathrm{P}<0.05)$, while the proportion of abdominal fat deposits increased significantly $(\mathrm{P}<0.05)$. Chromosome number of jade perch is 48 , karyo type is $2 \mathrm{sm}+2 \mathrm{~m}+44 \mathrm{t}$, and relative DNA content is $168.27 \pm 13.91$, which is significantly lower than that of another bass species, largemouth bass $(194.55 \pm 15.85)(P<0.05)$.

Keywords: Jade perch; Embryonic development; Morphological characters; Genetic characters; Gonadal development

\section{Introduction}

Jade perch (Scortum barcoo), also known as Barcoo grunter, is a fish species belonging to thegenus Scortum, the family Terapontidae and the order Perciformes. It originates from the Barcoo River of the Lake Eyre basin in central Australia and was introduced in China in 2001 [1]. The flesh of jade perch is firm and slightly flaky, sweet and succulent, without intermuscular bones, and isrich in nutrients, especially, highly unsaturated fatty acids. A study conducted by the Australian Common wealth Scientific and Industrial Research Organization (CSIRO) in 1998 indicated that among 200 sea food species tested, jade perch contained the highest level of omega-3, which was approximately 3 -fold that in Atlantic salmon and silver bass. Jade perch grow extremely fast andare highly suited for aquaculture in areas with a moderate subtropical to tropical climate. Under artificial aquaculture conditions, they can grow to food size in 6-10 months on a formulated diet. Recently, there is an increasing interest for species diversification to support the development of sustainable aquaculture. Fast-growing jade perch, which can be stocked at high densities in recirculating aquaculture systems (RAS) and feeds on grow-out diets with very low levels offishmeal and fish oil, is a promising candidate for aquaculture $[2,3]$. Currently, this species is being farmed in both intensive ponds and recirlulating systems not only in Australian, but also in China,
Malaysia, even in Belgium [4-6].Since the introduction of jade perch in China, domestic researchers have conducted several studies on the breeding and nutrition of this species. Chen et al. $[1,7]$ studied the artificial

propagation of jade perch and rearing of fry and fingerlings. Bao et al. [8] conducted a comparative analysis of the composition of typical nutrients (water, ash, crude protein, and crude fat), fatty acids,and amino acids in the muscle and viscera of jade perch, bass, and Chinese perch (Sinipercachuatsi), and Zhao et al. [9] assessed the nutritional value of jade perch. These results indicated that polyunsaturated fatty acids, sush as EPA and DHA, is extraordinarily high in jade perch. Feeding and nutrition requirements of jade perch were also investigated in other research. Van Hoestenberghe et al. [10] evaluated the effect of weaning age and the use of different sized Artemianauplii as first feed for jade perch. Alkhafaji et al. [6] determined the effect of feeding frequencies on the growth, plasma biochemistry, and liver glycogen of jade perch in a recirculating system. Song et al. [11] set up a study and it was also demonstrated that increasing lipid levels in fish diets was effective to improve protein utilization and decrease the nitrogen waste outputs and diet costs ofjade perch juveniles. Zhu et al. [12] characterized the bacterial community structure associated with filter material in the recirculating aquaculture system of jade perch. 
There are increasing research done with jade perch, however, studies on basic biological and genetic characteristics, such as cytogenetic data and DNA content, have not been reported. As jadeperch is native to Australia and was introduced in China for about 17 years now. Currently, a thorough knowledge and understanding of germ plasm characteristics of this introduced species isof great importance to the protection, improvement, and utilization of germplasm resources in China. Our previous research used mitochondrial D-loop sequences and simple sequence repeat(SSR) markers to analyze the genetic diversity in 4 reared jade perch populations in Guangdong, China. The results from the 2 methods showed that the 4 Guangdong populations had low genetic diversity and close phylogenetic relationships [13]. Therefore, there is a need to introduce theoriginal species in order to enrich the genetic diversity of the reared populations in China.

In this study, we aimed to assess relevant germplasm characteristics of jade perch through observation of gonadal development, embryonic development and morphological characters, determination of DNA content, and analysis of karyotype, using microscopy, assessment of morphological indicators, flow-cytometric analysis, and chromosome preparations from kidney cells of adult fish. From the perspective of basic biology and cytogenetics, a systematic observation and analysis of germplasm characteristics of jade perch not only provides a profound basis for large-scale artificial breeding and a reference for germplasm standards, but also offers guidance for future genetic breeding efforts.

\section{Materials and Methods}

\section{Experimental animals}

Jade perch used in this study were obtained from Liheng Aquaculture Farm (Xiqiao Town, Nanhai District, Foshan City, Guangdong Province, China). The parent used in artificial breeding had been domesticated and cultured for more than five years, weighed approximately $2 \mathrm{~kg}$ each, possessed a strong physique and high-quality traits, and were sexually mature. All applicable international, national, and institutional guidelines for the care and use of animals were followed.

\section{Artificial propagation and embryonic development in jade perch}

At a water temperature of $27.5 \pm 2^{\circ} \mathrm{C}$, two hormone injections were administered to the thoracic cavity of jade perch at $6 \mathrm{~h}$ intervals to induce spawning. For female fish, a mixture of human chorionic gonadotropin (HCG, 300-350IU• $\mathrm{kg}^{-1}$ ) and luteinizing hormone-releasing hormone (LRH-A2, 3-3.5 $\mu \mathrm{g} \mathrm{kg}^{-1}$ ) was administered during the first injection, while for the second injection was a mixture of domperidone (DOM, $\left.8 \mathrm{mg} \bullet \mathrm{kg}^{-1}\right)$ and LRH-A2 $\left(8 \mu \mathrm{g} \bullet \mathrm{kg}^{-1}\right)$. When the second injection was administered to female fish, a quarter-dose was administered to male fish as well. Subsequently, male and female fish were artificially paired for spawning and fertilization, with afemale to male ratio of
1:1. The zygotes were placed in a circular pond for incubation. Through out the incubation period, a portion of zygotes were periodically removed and placed in petri dishes for observation. Observations, image acquisition, and recording of the process and timeline of embryonic development were carried out using a ZEISS Stereo Discovery V8 microscope.

\section{Measurements of morphological characters}

Morphological characters of jade perch at different developmental stages were measured inaccordance with the People's Republic of China Standard on the Inspection of Germplasm for Cultured Fishes - Part 3: Measurement of Characters (GB/T 18654.3-2008). Specifically, 30 two-monthold jade perch and 30 six-month-old jade perch were selected for quantification of seven countable characters (lateral line scales, scales above the lateral line, scales below the lateral line, dorsal fin rays, pectoral fin rays, abdominal fin rays, anal fin rays) as well as determination of nine measurable characters (total length, body length, head length, caudal peduncle length, head depth, body depth, caudal peduncle depth, body weight, and weight of fat deposits). Subsequently, the following ratios were calculated: whole length/body length, body length/head length, body

length/body depth, head length/head depth, caudal peduncle length/caudal peduncle depth, body depth/head depth, and weight of fat deposits/body weight. The data were analyzed by analysis of variance using SPSS Statistics 17.0, with $\mathrm{P}<0.05$ regarded as significant.

\section{Analysis of blood DNA content}

Ten six-month-old jade perch were randomly selected, and $10 \mu \mathrm{L}$ of blood was collected from the caudal veins of each fish. Then, $100 \mu \mathrm{L}$ of PBS was added and the mixture was homogenized by shaking to form a cell suspension. $1 \mathrm{~mL}$ of $70 \%$ ethanol was added for cell fixation at $4^{\circ} \mathrm{C}$ for $1 \mathrm{~h}$. Subsequently, the suspension was centrifuged at $300 \times \mathrm{g}$ for $5 \mathrm{~min}$ at room temperature, and thesupernatant was removed. The remaining liquid (approximately $50 \mu \mathrm{L}$ ) was treated with $30 \mu \mathrm{L}$ of $1 \mathrm{mg} / \mathrm{mL}$ propidium iodide (PI) staining solution (CyStain PI Absolute, Partec). Then, the mixture was diluted to $500 \mu \mathrm{L}$ with PBS and placed in a dark room to facilitate staining in the dark for $1 \mathrm{~h}$.

After staining, the sample was filtered through a Partec filter and analyzed using a flow cytometer(Cell Lab Quanta, Beckman Coulter). Another common freshwater bass species, the large mouth bass (Micropterussalmoides), was used as a control. The DNA contents of all samples were compared using the $\chi 2$ test with Yates' continuity correction.

\section{Chromosome preparations of kidney cells}

Chromosome preparations of kidney cells were carried out using the protocal reported by Liuet al. [14], with minor modifications. Three six-month-old jade perch were randomly selected and injected with $8-10 \mu \mathrm{g} / \mathrm{g}$ of phytohemagglutinin 
(PHA) + 2-4 $\mu \mathrm{g} / \mathrm{g}$ of colchicine (Sigma). 2-3h later, head kidney of the fish was dissected, cut into fragments after removal of blood clots and connective tissues, and transferred to a centrifuge tube. A small volume of $0.8 \%$ saline was added, the mixture was pipetted up and down to generate a flocculated suspension, and saline was added again to obtain a final volume of $10 \mathrm{~mL}$. The suspension was placed aside for precipitation, then centrifuged at a speed of 1000-1500rpm. After discarding the supernatant, the remaining liquid was added to $10 \mathrm{~mL}$ of hypotonic solution containing $0.0373 \mathrm{M} \mathrm{KCl}$ and subjected to hypo-osmosisat room temperature for $45-60 \mathrm{~min}$. Then, the sample was centrifuged at 1000-1500rpm, the hypotonic solution was removed, and the remaining liquid was fixed using a pre-chilled mixture of methanol and acetic acid $(3: 1)$ for $30 \mathrm{~min}$. The fixation process was repeated 2-3 times. Then, the cell suspension was dripped from a height of approximately $20 \mathrm{~cm}$ onto a pre-chilled glass slide.

The slide was rapidly baked, and then allowed to air-dry. Lastly, the chromosome preparation was stained using a Giemsa stain solution and rinsed approximately 30 minutes later. After removal of the stain solution using water, the preparations were observed and 50 mitotic metaphase spreadsin each sample were

Table 1:Time table of embryonic development of jade perch. photographed and analyzed under a Leica DM2500 microscope.

\section{Histological observation on the gonads}

Each three of females and males at six-month-old and fouryear-old were randomly selected for histological observations on the gonad structure by preparing paraffin sections. The gonad of each fish was fixed in Bouin's solution, embedded in paraffin, then cut into sections with a Leica RM2016 and then stained with hematoxylin and eosin. Tissue sections were observed and photographed with a Leica DM2500. The gonad development of each sample was identified according to the standards for largemouth bass [15].

\section{Results}

\section{Early embryonic development in jade perch}

It was observed that zygotes of jade perch are transparent, pelagic, and swell upon absorption of water to up to $2.1 \pm 0.3 \mathrm{~mm}$ in diameter. Each zygote typically contained one oil globule ( $\sim 1 / 4$ of the diameter of the zygote), with a minority of zygotes containing two to three oil globules. At awater temperature of $27.5 \pm 2^{\circ} \mathrm{C}$, jade perch fry hatched after $21-26 \mathrm{~h}$ of normal embryonic development. Table 1 and Figure 1 show the time and various stages of embryonic development of jade perch zygotes.

\begin{tabular}{|c|c|c|}
\hline Embryonic Period & Embryonic Stage & Time After Fertilization \\
\hline Fertilization & zygote & 0.00 \\
\hline \multirow{6}{*}{ Cleavage } & blastodisc formation & $17 \mathrm{~min} \sim 33 \mathrm{~min}$ \\
\hline & 2-cell & $34 \mathrm{~min} \sim 43 \mathrm{~min}$ \\
\hline & 4-cell & $45 \mathrm{~min} \sim 56 \mathrm{~min}$ \\
\hline & 8-cell & $56 \mathrm{~min} \sim 1 \mathrm{~h} 8 \mathrm{~min}$ \\
\hline & 16-cell & $1 \mathrm{~h} 10 \mathrm{~min} \sim 1 \mathrm{~h} 23 \mathrm{~min}$ \\
\hline & multi-cell & $2 \mathrm{~h} 22 \mathrm{~min} \sim 2 \mathrm{~h} 40 \mathrm{~min}$ \\
\hline \multirow{3}{*}{ Blastula } & high blastula & $3 \mathrm{~h} 20 \mathrm{~min} \sim 4 \mathrm{~h}$ \\
\hline & mid blastula & $4 \mathrm{~h} 4 \mathrm{~min} \sim 4 \mathrm{~h} 40 \mathrm{~min}$ \\
\hline & low blastula & $4 \mathrm{~h} 44 \mathrm{~min} \sim 5 \mathrm{~h} 30 \mathrm{~min}$ \\
\hline \multirow{3}{*}{ Gastrula } & early gastrula & $5 \mathrm{~h} 37 \mathrm{~min} \sim 6 \mathrm{~h} 30 \mathrm{~min}$ \\
\hline & mid gastrula & $6 \mathrm{~h} 49 \mathrm{~min} \sim 8 \mathrm{~h}$ \\
\hline & late gastrula & $8 \mathrm{~h} \sim 8 \mathrm{~h} 45 \mathrm{~min}$ \\
\hline \multirow{3}{*}{ Neurula } & blastopore closure & $8 \mathrm{~h} 47 \mathrm{~min} \sim 10 \mathrm{~h} 15 \mathrm{~min}$ \\
\hline & early neurula & $10 \mathrm{~h} 21 \mathrm{~min} \sim 11 \mathrm{~h} 24 \mathrm{~min}$ \\
\hline & embryoid body formation & $11 \mathrm{~h} 24 \mathrm{~min} \sim 13 \mathrm{~h} 26 \mathrm{~min}$ \\
\hline \multirow{4}{*}{ Organogenesis } & sarcomere emergence & $13 \mathrm{~h} 30 \mathrm{~min} \sim 13 \mathrm{~h} 59 \mathrm{~min}$ \\
\hline & tail bud & $13 \mathrm{~h} 59 \mathrm{~min} \sim 16 \mathrm{~h} 35 \mathrm{~min}$ \\
\hline & optic vesicle formation & $16 \mathrm{~h} 35 \mathrm{~min} \sim 19 \mathrm{~h} 04 \mathrm{~min}$ \\
\hline & muscular contraction & $19 \mathrm{~h} 04 \mathrm{~min} \sim 20 \mathrm{~h} 45 \mathrm{~min}$ \\
\hline Hatching & hatching & $21 \mathrm{~h} \sim 26 \mathrm{~h}$ \\
\hline
\end{tabular}



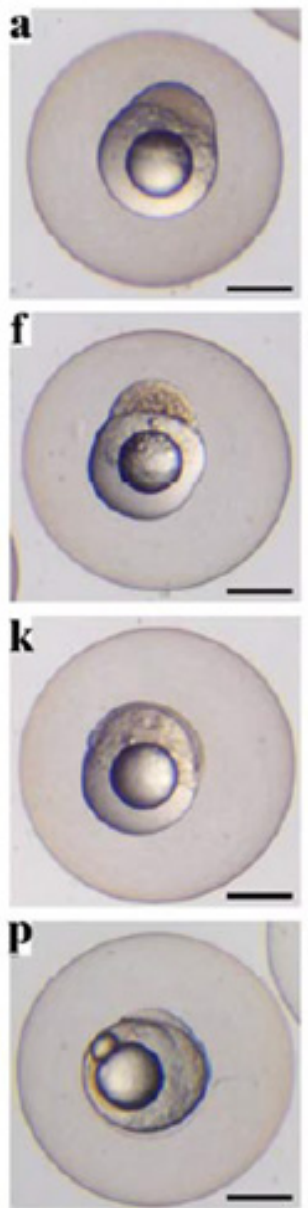
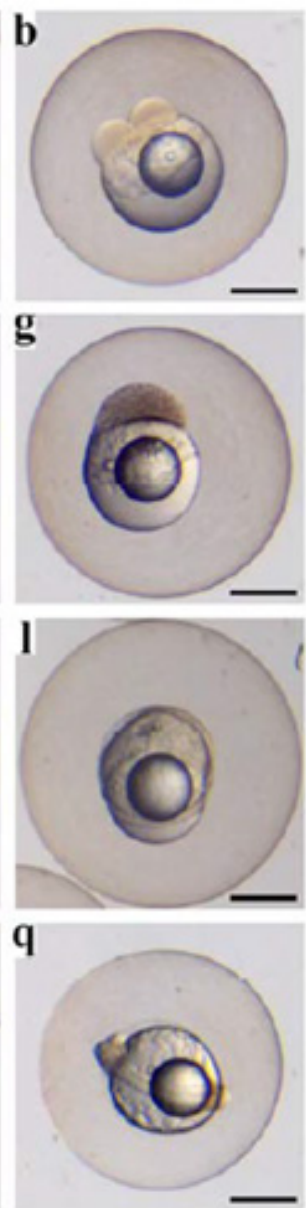
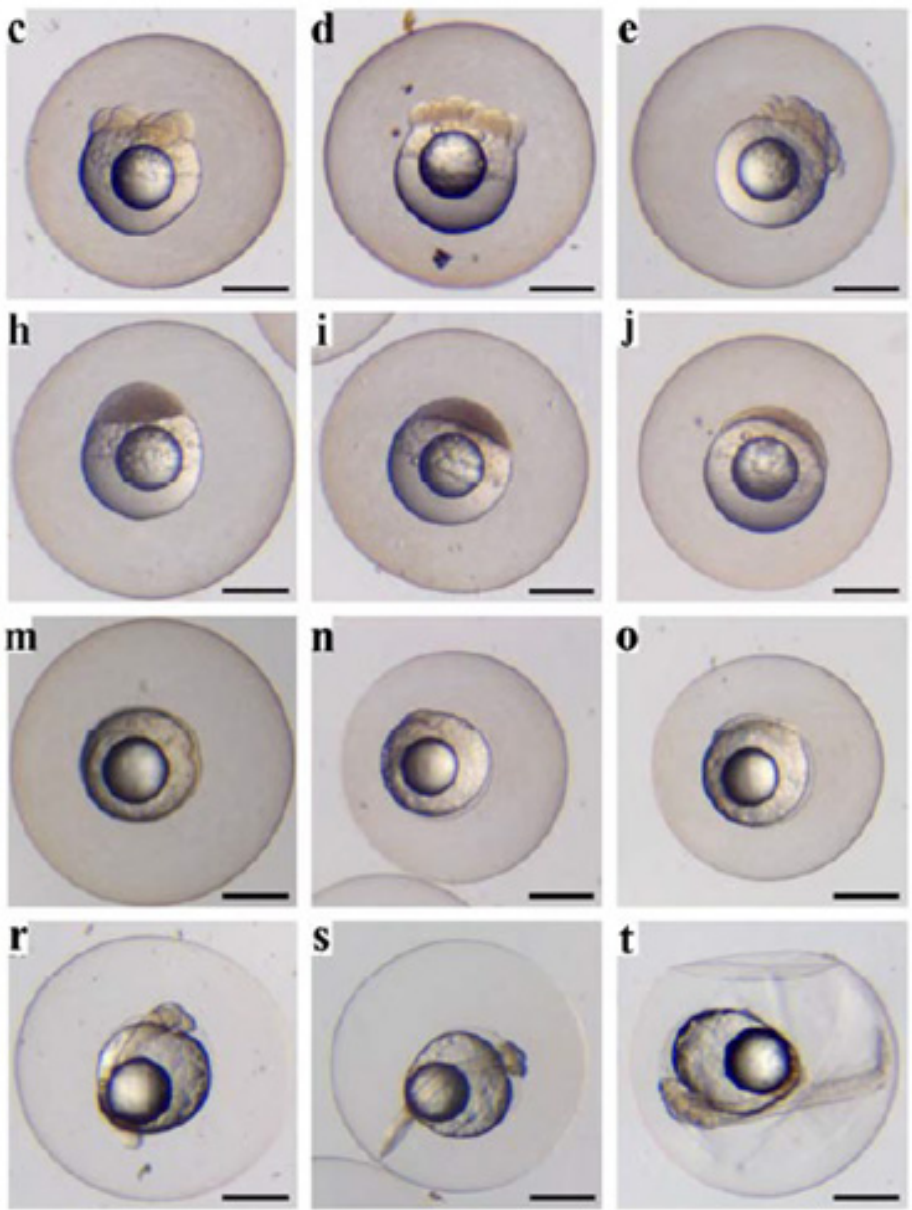

Figure 1: Embryonic development of jade perch. a: Blastodisc formation. b: Two-cell stage; c: Four-cellstage; d: Eight-cell stage; e: Sixteencell stage; f: Multi-cell stage; g: High blastula stage; h: Midblastula stage; i: Late blastula stage; j: Early gastrula stage; k: Mid gastrula stage; I: Late gastrulastage; m: Blastopore closure stage; $n$ : Early neurula stage; o: Embryoid body formation stage; $p: O r g a n o g e n e s i s$ and sarcomere emergence; q: Tail bud stage; r: Optic vesicle formation stage; s:Muscular contraction stage; t: Hatching. Bar=1mm.

Figure 1 shows various stages of embryonic development of jade perch zygotes. After fertilization, the zygote membrane swells upon absorption of water, and as early as 17-33min after fertilization, the zygote cytoplasm is concentrated at the animal pole and gradually bulges to form an embryonicdisc (Figure 1a). Subsequently, the zygotes go through the cell division stage at 2h40min after fertilization (Figure 1b-1f), then, blastula stage at $5 \mathrm{~h} 30 \mathrm{~min}$ (Figure $1 \mathrm{~g}$-1i), gastrula stage at $8 \mathrm{~h} 45 \mathrm{~min}$ (Figure $1 \mathrm{j}$ 11), neurula stage at $13 \mathrm{~h} 26 \mathrm{~min}$ (Figure $1 \mathrm{~m}-1 \mathrm{o}$ ), organogenesis stage at $20 \mathrm{~h} 45 \mathrm{~min}$ (Figure $1 \mathrm{p}-1 \mathrm{~s}$ ) and so on, until hatching at 21h-26h after fertilization (Figure $1 \mathrm{t}$ ).

\section{Morphological characters of jade perch}

Jade perch has a spindle-shaped body, and its back is covered with brilliant green scales, with typically one or more black spots on both sides of the body (Figure 2). In this study, the mean weights of two- and six-month-old jade perch were $88.67 \pm 31.76 \mathrm{~g}$ (Figure 2a) and $338.57 \pm 72.50$ g (Figure 2b), respectively. As shown in Table 2, no significant differences were observed in countable characters of jade perch in different developmental stages $(\mathrm{P}>0.05)$. Number of countable characters were as follows: 13-14 dorsal fin spines, 11-13 dorsal fin rays; 14-16 pectoral fin rays; 1 abdominal finspine, 5 abdominal fin rays; 3 anal fin spines, 8-9 anal fin rays; 65-78 lateral line scales, 13-15scales above the lateral line, and 24-27 scales below the lateral line. Analysis of the ratios of measurable characters indicated that the body length/head length, body depth/head depth, and weight of fat deposits/body weight of two-month-old jade perch were $4.27 \pm 0.48,1.74 \pm 0.11$, and $0.06 \pm 0.02$, respectively, which were significantly lower than those of six-month-old perch, $5.19 \pm 0.53,2.13 \pm 0.17,0.14 \pm 0.01$, respectively $(\mathrm{P}<0.05)$ (Table $3)$. No significant differences $(\mathrm{P}>0.05)$ were found for the other ratios, i.e., total length/body length, body length/body depth, head length/head depth, and caudal peduncle length/caudal peduncle. Compared with two-month-old fish, the proportion of the head with respect to the body was significantly decreased $(\mathrm{P}<0.05)$, while the proportion of fat deposits was significantly increased in six-month-old fish $(\mathrm{P}<0.05)$. 

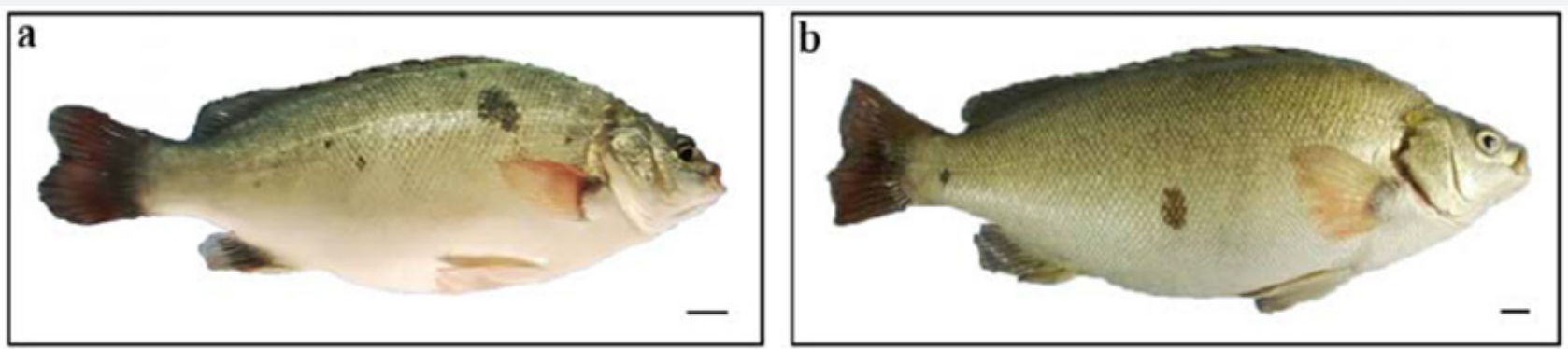

Figure 2: Appearance of jade perch. a: Two-month-old; b: Six-month-old. Bar=1cm.

Table 2: Countable characters of jade perch. Roman numerals represent hard fin spines and Arabic numerals represent soft fin rays.

\begin{tabular}{|c|c|c|c|c|c|c|c|}
\hline $\begin{array}{c}\text { Countable } \\
\text { Characters }\end{array}$ & $\begin{array}{c}\text { Lateral Line } \\
\text { Scales }\end{array}$ & $\begin{array}{c}\text { Scales Above the } \\
\text { Lateral Line }\end{array}$ & $\begin{array}{c}\text { Scales Below the } \\
\text { Lateral Line }\end{array}$ & $\begin{array}{c}\text { Dorsal Fin } \\
\text { Rays }\end{array}$ & $\begin{array}{c}\text { Pectoral Fin } \\
\text { Rays }\end{array}$ & $\begin{array}{c}\text { Abdominal } \\
\text { Fin Rays }\end{array}$ & $\begin{array}{c}\text { Anal Fin } \\
\text { Rays }\end{array}$ \\
\hline Two-month-old & $65 \sim 78$ & $13 \sim 14$ & $24 \sim 26$ & $\begin{array}{c}\text { XIII XIV } \\
+11 \sim 13\end{array}$ & $15 \sim 16$ & I+5 & III +8 9 \\
\hline Six-month-old & $74 \sim 77$ & $13 \sim 15$ & $25 \sim 27$ & XIII +10 12 & $14 \sim 16$ & I+5 & III +8 9 \\
\hline
\end{tabular}

Table 3: Measurable characters of jade perch.

\begin{tabular}{|c|c|c|c|c|c|c|c|c|c|}
\hline $\begin{array}{c}\text { Measurable } \\
\text { Characters }\end{array}$ & $\begin{array}{c}\text { Whole } \\
\text { Length/ } \\
\text { Body } \\
\text { Length }\end{array}$ & $\begin{array}{c}\text { Body } \\
\text { Length/ } \\
\text { Head } \\
\text { Length }\end{array}$ & $\begin{array}{c}\text { Body } \\
\text { Length/ } \\
\text { Body } \\
\text { Depth }\end{array}$ & $\begin{array}{c}\text { Head } \\
\text { Length/ } \\
\text { Head } \\
\text { Depth }\end{array}$ & $\begin{array}{c}\text { Head } \\
\text { Length/ } \\
\text { Head } \\
\text { Depth }\end{array}$ & $\begin{array}{c}\text { Caudal } \\
\text { Peduncle } \\
\text { Length/ } \\
\text { Caudal } \\
\text { Peduncle }\end{array}$ & Body Weight & $\begin{array}{c}\text { Weight } \\
\text { of Fat } \\
\text { Deposits }\end{array}$ & $\begin{array}{c}\text { Weight of Fat } \\
\text { Deposits/ } \\
\text { Body Weight }\end{array}$ \\
\hline $\begin{array}{c}\text { two-month- } \\
\text { old }\end{array}$ & $1.16 \pm 0.02$ & $4.27 \pm 0.48$ & $2.72 \pm 0.16$ & $1.11 \pm 0.12$ & $1.74 \pm 0.11$ & $1.14 \pm 0.21$ & $88.67 \pm 31.76$ & $5.67 \pm 1.51$ & $0.06 \pm 0.02$ \\
\hline $\begin{array}{c}\text { six-month- } \\
\text { old }\end{array}$ & $1.14 \pm 0.03$ & $5.19 \pm 0.53$ & $2.64 \pm 0.19$ & $1.10 \pm 0.18$ & $2.13 \pm 0.17$ & $1.06 \pm 0.11$ & $338.57 \pm 72.50$ & $46.43 \pm 10.45$ & $0.14 \pm 0.01$ \\
\hline
\end{tabular}

\section{DNA Content}

Mean DNA content in 5000 blood cells of each sample was determined, using largemouth bassas a control. As shown in Figure 3, for largemouth bass, normal diploid cells accounted for $85.18 \%$ of cells, and the mean DNA content was 175.4 (Figure 3a), while in jade perch, normal diploid cells accounted for 84.4\% of cells, and the mean DNA content was 154.7 (Figure 3b). Statistical analysis of data from 10 samples revealed that the DNA content in jade perch $(168.27 \pm 13.91)$ was significantly lower than that in largemouth bass $(194.55 \pm 15.85)(\mathrm{P}<0.05)$.
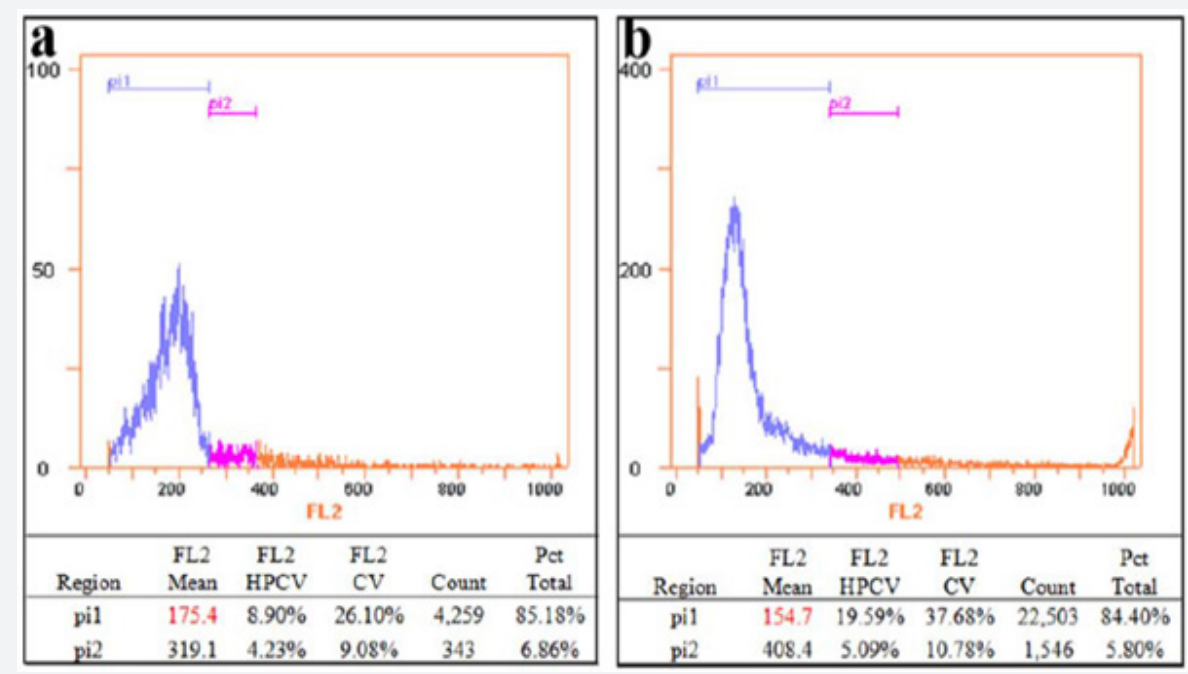

Figure 3: Flow cytometry of jade perch. a: DNA content of largemouth bass (control); 516 b: DNA content of 517 jade perch. 


\section{Karyotypeanalysis}

From the chromosome preparations from kidney cells, 50 mitotic metaphase spreads with clear morphologies and a good degree of dispersion were selected for analysis. Lengths of long and short arms of each chromosome were measured with an ocular micrometer, and the arm ratios ( $\mathrm{r}=$ length of long arm/length of short arm) were calculated for karyotype analysis. Chromosomes were then classified according to the classification standard developed by Levan et al. [14], i.e., $r=$ 1.0-1.7: metacentric $(\mathrm{m}), \mathrm{r}=1.8-3.0$ : submetacentric $(\mathrm{sm}), \mathrm{r}=$ 3.1-7.0: subtelocentric (st), $r=7.1-\infty$ : telocentric $(\mathrm{t})$. Karyotype analysis showed that jade perch has 48chromosomes (Figure 4a), including 1 pair of submetacentric chromosomes, 1 pair of metacentric chromosomes, and 22 pairs of telocentric chromosomes, and the karyotype formula is $2 \mathrm{sm}+2 \mathrm{~m}+44 \mathrm{t}$ in somatic cells (Figure 4b).

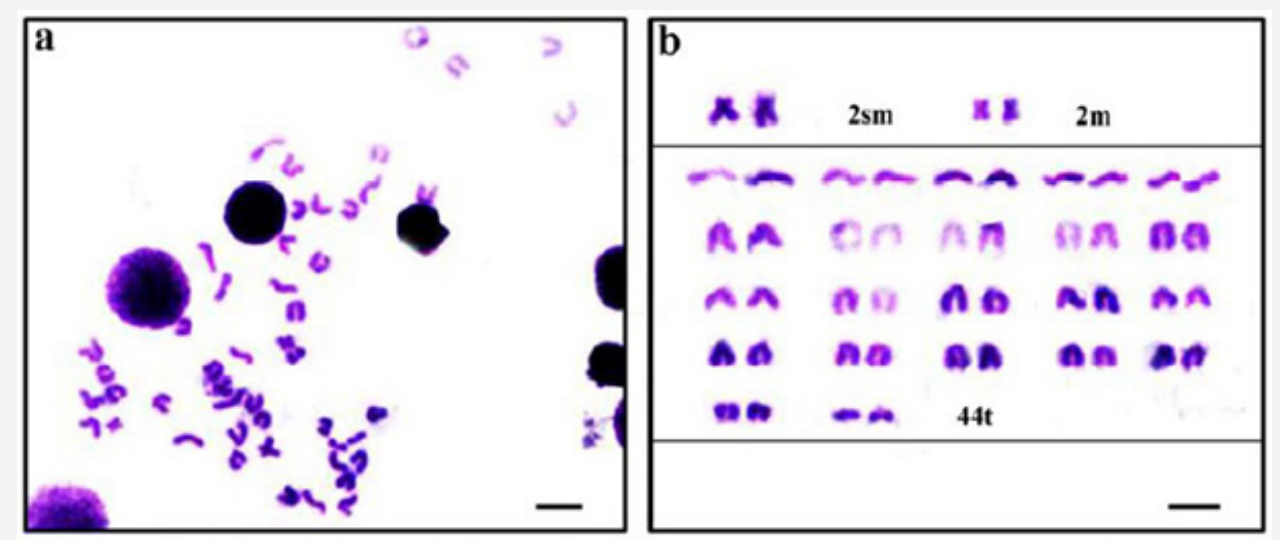

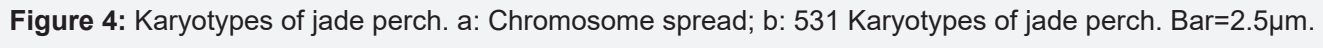

\section{Analysis of gonadal development}

Figure 5 presents gonad micro structure of food-sized jade perch at about six-month-old. When it reach the market size, testes of jade perch did not form typical seminiferous tubules, remained primitive spermatogonium without proliferation and differentiation, and almost no spermatocytes were found in them (Figure 5a). Ovaries developed normally in which the oogonia proliferated massively and have developed into oocytes in phase II (Figure 5b).

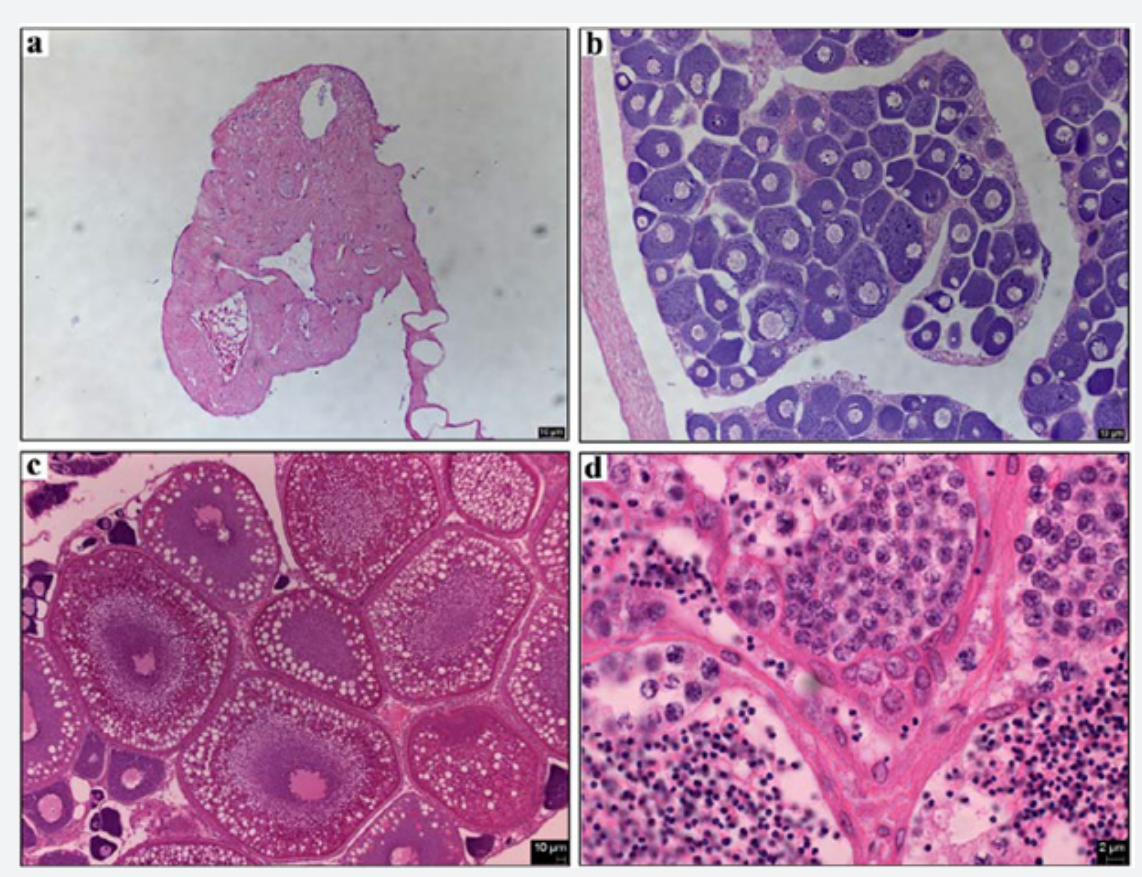

Figure 5: Gonad microstructure of jade perch. a: Testis of jade perch at six-548 month-old. Bar=10 $\mu \mathrm{m}$; b: Ovary549 of jade perch at at sixmonth-old. Bar $=10 \mu \mathrm{m} ; \mathrm{c}$ : Testis of jade perch at four-year-old. Bar=10 $\mu \mathrm{m} ; \mathrm{d}$ : Ovary550 of jade perch at at four-year-old. Bar $=2 \mu \mathrm{m}$. 
Normal ovaries in female jade perch at four-year-old were found oblong and soft, which almost filled the abdominal cavity. Section observation showed that the ovaries of four-year-old jade perch were in III to IV-stage and the oocytes were full of yolk (Figure 5c). Testes of jade perch were milk-white, bilateral symmetry and well developed. Observing the microstructure of the testes of jadeperch, we found groups of spermatogonia, primary spermatocytes, secondary spermatocytes, spermatids and numerous mature spermatozoa, were filled in seminiferous tubules (Figure $5 \mathrm{~d}$ ). Semen could be squeezed out of these testes of jade perch at four-year-old.

\section{Discussion}

Jade perch possesses several advantages, including sweet and succulent flesh, rapid growth, and good suitability for industrial aquaculture. It deserves to be promoted as a profitable commercial fresh water species. In this study, analysis of gonadal development indicated that jadeperch get to sexually mature at four-year-old, when the ovaries possess partial oocytes in phase IV suitable for artificial spawing, and mature sperm could be squeezed out of testes in jade perch.

Embryonic development in jade perch was evaluated. It was found that upon absorption of water, the zygotes of jade perch could swell to a average diameter of $2.1 \mathrm{~mm}$, which is much larger thanthe zygote diameters of some other species of order Perciformes, e.g., Japanese seabass (Lateolabrax japonicus): $1.35-1.44 \mathrm{~mm}$ [16], and largemouth bass: $1.32-1.40 \mathrm{~mm}$ [17], however, significantly smaller than that of Striped Bass, which is 2.45-3.14mm [18]. It was observed that ata water temperature of $27.5 \pm 2{ }^{\circ} \mathrm{C}$, zygotes of jade perch hatched within $21-26 \mathrm{~h}$ of fertilization.

For Japanese seabass, the optimum breeding temperature is $12-14^{\circ} \mathrm{C}$, and within this temperature range, embryonic development takes $\sim 85 \mathrm{~h}$. For large mouth bass, when breeding is carried out ina temperature range of $18-20^{\circ} \mathrm{C}$, embryonic development takes $\sim 58 \mathrm{~h}$. The process of embryonic development in jade perch is similar to that of seabass [19] and large mouth bass [20], i.e., after the cleavage, blastula, and gastrula stages, the embryoid body is formed, sarcomeres and tail budsemerge, and various organs are formed; subsequently, muscular contraction occurs, and the heartbeat and blood circulation are established before hatching takes place. Body weight, which is correlated with morphological traits at different levels, is an important target in the selective breeding of growth traits in fish species. For instance, in black bream, whichis another member of the family Terapontidae, head depth, body width, caudal peduncle depth, and body length are four major morphological characters that affect body weight in the adult, with headdepth having the most significant direct impact (P2 = 0.360) [21]. In largemouth bass, body width, body length, and interocular distance are strongly positively correlated with body weight, and arethe major morphological characters that directly or indirectly affect body weight [22]. In addition, differences in developmental stage, habitat or even stress state may cause differences in morphological characteristics in individuals. For instance, the contribution rates of total length tomorphological characters in four- and six-month-old largemouth bass are $92.29 \%$ and $86.40 \%$,

respectively; therefore, this indicator can be used as a selection criterion for morphological characters in this species. The contribution rates of total length/body depth and caudal peduncle length/caudal peduncle depth to body size in fourmonth-old largemouth bass are $65.94 \%$ and $34.06 \%$, respectively, while the contribution rates in six-month-old largemouth bass are $69.08 \%$ and $30.92 \%$, respectively; therefore, these two indicators can be used as selection criteria for bodysize in largemouth bass [23]. In this study, conventional morphological methods were used to compare the physical appearances of two- and six-month-old jade perch, and it was found that the proportion of the head with respect to the body was significantly decreased $(\mathrm{P}<0.05)$, and that offat deposits was significantly increased in six-month-old fish $(\mathrm{P}<0.05)$, whereas differences inother characters were not significant $(\mathrm{P}<0.05)$. This suggests that body length/head length andbody depth/head depth are strongly corrlated with body weight in jade perch; therefore, Using these parameters as indicators of growth traits for selective breeding may be considered. In six-month-old jade perch, the proportion of abdonimal fat deposits to body weight was as high as $14 \%$, which is close to the average content of fat in whole cutlets of some highly valued fish species. Fat percentage of a cutlet is $14.6 \%$ in Atlantic halibut (Hippoglossushippoglossus L.), which is a highly valued fish species [24]. Large fat deposits in the abdomen of adult jade perch may be a species-specific characteristic. Further studies are required to determine if fat content in jade perch can beregulated through changes in nutrient ratios of the feed and breeding methods, and to investigate methods for the effective exploitation and utilization of unsaturated fatty acids in jade perch. Chromosomes are carriers of genetic material, and chromosome evolution is closely linked tospecies evolution. Changes in chromosome number and structure may lead to mutations in germplasm characteristics or even the emergence of new species. Knowledge of karyotype characteristics allows a better understanding of a species' phylogenetic status. In general, with in specific taxonomic categories, primitive groups have more telocentric chromosomes, while specialized groups have more metacentric or submetacentric chromosomes [25]. More primitivefish species have a higher number of telocentric and subtelocentric chromosomes, fewer or nometacentric and submetacentric chromosomes, and a lower total number of chromosome arms[26]. The karyotype formula of jade perch is $2 \mathrm{sm}+2 \mathrm{~m}+$ $44 \mathrm{t}$. The karyotype formulae of some other species of the order Perciformes are as follows: black bream: $4 m+44 t$ [26], Japanese seabass: 48t, Queensland grouper (Promicropslanceolatus): 4st 
$+44 \mathrm{t}$, and brown-marbled grouper (Epinephelus fuscoguttatus): $2 \mathrm{sm}+46 \mathrm{t}$ [27]. It can be deduced that among fish species of the order Perciformes, jade perch is a specialized species and has a higher evolutionary status. Largemouth bass has achromosome number of 46 , with karyotype formula $2 m+2 s t$ + 42t (Germplasm Standard for Large mouth Bass, GB210452007), and a DNA content of $194.55 \pm 15.85$, which is significantly higher than the DNA content in jade perch $(168.27 \pm 13.91)$ $(\mathrm{P}<0.05)$. Compared with large mouth bass, jade perch has more chromosomes but lower DNA content; therefore, we can preliminarily deduce that jade perch has a smaller genome and low redundancy in genomic information, which facilitates future measurement and calculation of jade perch genome size as well as the implementation of transcriptome and genome sequencing projects.

Chromosome number and karyotype also serve as a biological basis for distant hybridization. Hence, it is worth while to study these aspects in further detail. For instance, a study on the distanthy bridization between parental species with the same chromosome numbers and between parental species with different chromosome numbers revealed that distant hybridization could result in off spring with different ploidy levels [28]. On the basis of karyotype analysis, if techniques such as G-banding and fluorescent in-situ hybridization can be integrated with studies of chromosome evolution in fish species, a better understanding of the genetic composition and biological development can be attained. In addition, such studies will facilitate the identification of congeneric species and phylogenetic studies, and are of great significance for breeding and hybridization.

\section{Conclusion}

Jade perch, native in Australia, becomes a promising fresh water bass species for aquaculture in China recently. Their populations in southern China have been identified with low degree of genetic diversity and differentiation. To protect, improve and utilize the germplasm resources of this introduced species, knowledge about the biological characteristics, such as morphological and cytogenetical data, is necessary to be known.

Jade perch zygotes hatched 21-26h after fertilization at a water temperature of $27.5 \pm 2{ }^{\circ} \mathrm{C}$. The proportion of the head with respect to the body decreased significantly between twoand six-month-old jade perch $(\mathrm{P}<0.05)$, while the proportion of abdominal fat deposits increased significantly $(\mathrm{P}<0.05)$. Body length/head length and body depth/head depth ratios could serve aspotential indicators of growth traits for selective breeding. Based on chromosome number and DNA content analysis, we deduced that jade perch has a relatively small genome and low redundancy in genomic information, which facilitates future measurements and calculations of jade perch genome size as well as the implementation of transcriptome and genome sequencing projects.

\section{Ethical Approval}

All applicable international, national, and institutional guidelines for the care and use of animals were followed, and this article does not contain studies with human participants performed by any of the authors.

\section{Acknowledgements}

We are most grateful to the following persons for collecting the jade perch samples: LixiangZhao, Hailin Sun and Zhuoming Huang. This research was supported by Central Public-interest Scientific Institution Basal Research Fund, CAFS (NO. 2017HYXKQ0208), China Agriculture Research System (CARS-46), Special Fund for Scientific Research in Public Welfare and Capacity Building of Guangdong Province (NO. 2017A030303002) and the Natural Science Foundation of Guangdong Province (NO. 2015A030310154).

\section{Conflict of Interest}

All the authors declare that they have no conflict of interest.

\section{References}

1. Chen KC, Zhu XP, Du HJ, Xie G, Liu YH, et al. (2007) Effects of temperature and salinity on the embryonic development of jade perch scortum barcoo. J Fish Sci China 14(6): 1032-1037.

2. Luo G, Zhang N, Tan H (2012) Effect of low salinity on jade perch scortum barcoo performance in a recirculating aquaculture system. $\mathrm{N}$ Am J Aquacult 74(3): 395-399.

3. Van Hoestenberghe S, Roelants I, Vermeulen D, Goddeeris BM (2013) Total replacement of fish oil with vegetable oils in the diet of juvenile Jade perch scortum barcoo reared in recirculating aquaculture systems. J Agric Sci Tech B 3: 385-398.

4. Mok WJ, Senoo S, Itoh T, Tsukamasa Y, Kawasaki KI, et al. (2012) Assessment of concentrations of toxic elements in aquaculture food products in malaysia. Food Chem 133(4): 1326-1332.

5. Liu L, Li YW, He RZ, Xiao XX, Zhang X, et al. (2014) Outbreak of streptococcus agalactiae infection in barcoo grunter, scortum barcoo (mcculloch \& waite), in an intensive fish farm in china. J Fish Dis 37(12): 1067-1072.

6. Alkhafaji FM, Romano N, Amin SMN, Fadel AHI, Ebrahimi M, et al. (2017) Effects of feeding frequencies on the growth, plasma biochemistry, and liver glycogen of jade perch scortum barcoo in a recirculating system. $\mathrm{N}$ Am J Aquacult 79(3): 216-223.

7. Chen KC, Zhu XP, Zhao J (2010) Techniques on artificial propagation, seed rearing and cultivation techniques of jade perch (Scortum bacoo). China Fish 3: 43-46.

8. Bao D, Tao NP, Ding ZP (2006) Comparative analysis of nutritional composition on scortum barcoo, lateolabrax japonicus and siniperca chuatsi. J Shanghai Fish U 15(1): 123-127.

9. Zhao J, Chen KC, Zhu XP, Zheng GM, Chen YL, Pan DB (2011) Analysis and evaluation of muscle nutrients in jade perch scortum barcoo. J of Dalian Ocean U 26(1): 93-96.

10. Van Hoestenberghe, S, Wille M, Swaef ED, Goddeeris, BM, Nevejan N (2015) Effect of weaning age and the use of different sized Artemia nauplii as first feed for jade perch scortum barcoo. Aquacult Int 23(6): 1539-1552.

11. Song LP, An L, Zhu YA, Li X, Wang AY (2009) Effects of dietary lipids on growth and feed utilization of jade perch, scortum barcoo. J World Aquacult Soc 40(2): 266-273. 
12. Zhu P, Ye Y, Pei F, Lu K (2012) Characterizing the structural diversity of a bacterial community associated with filter materials in recirculating aquaculture systems of, scortum barcoo. Can J of Microbiol 58(3): 303310.

13. Lixiang Zhao, Junjian Dong, Chengfei Sun, Yuanyuan Tian, Jie Hu, et al. (2018) Analysis of genetic diversity in reared jade perch populations using a combination of mitochondrial D-loop sequences and simple sequence repeat markers. Freshwater Fish 6 (in press).

14. Liu SJ, Liu Y, Zhou GJ, Zhang XJ, Luo C, et al. (2001) The formation of tetraploid stocks of red crucian carp $\times$ common carp hybrids as an effect of interspecific hybridization. Aquaculture 192(2-4): 171-186.

15. Strauss K, Vicari S, Valeri G, D’Elia L, Arima S, et al. (2010) Gonadal maturation and its induction in largemouth bass, micropterus salmoides. Suisanzoshoku 39(2): 343-351.

16. Mito S (1957) On the egg development and larvae of a japanese sea bass, lateolabrax japonicus (cuvier). Sci Bull Fac Agr Kyushu U 16.

17. Merriner J (1971) Egg size as a factor in intergeneric hybrid success of centrarchids. T Am Fish Soc 100(1): 29-32.

18. Steven S, Brian B (1999) Effects of increasing water hardness on egg diameter and hatch rates of striped bass eggs. N Am J Aquacult 61(3): 263-265.

19. Cucchi P, Sucré E, Santos R, Leclère J, Charmantier G et al. (2012) Embryonic development of the sea bass dicentrarchus labrax. Helgoland Mar Res 66(2): 199-209.

20. Philipp DP, Kaminski C, Whitt GS (1985) A comparison of the embryonic development of northern, Florida and reciprocal F1 hybrid largemouth bass in different thermal environments. Illinois Nat Hist Surv Bull 334 261-273.
21. Yang LD, Huang C, Shu H, Liu ZQ, Sun GB, et al. (2013) Relationship between morphometric attributes and body weight of hephaestus fuliginosus. Guangdong Agr Sci 10: 117-120.

22. He XY, Liu XL, Bai JJ, Li SJ, Fan JJ (2009) Mathematical analysis of effects of morphometric attribute on body weight of largemouth bass (micropterus salmoides). J Fish China 33(4): 597-603.

23. Li R, Bai JJ, Li SJ, Ye X (2011) Correlation and principal components analysis of morphological traits of micropterus salmoides family at early age. Mar Fish 33(3): 282-288.

24. Kolstad K, Vegusdal A, Baeverfjord G, Einen O (2004) Quantification of fat deposits and fat distribution in atlantic halibut (hippoglossus hippoglossus, L.) using computerised x-ray tomography (CT). Aquaculture 229(1-4): 255-264.

25. Campos H, Arratia G, Cuevas C (2010) Karyotypes of the most primitive catfishes (teleostei: siluriformas: diplomystidae). J Zool Syst Evol Res 35(3): 113-119.

26. Meng QL, Li AN, Wang CW, Xian LI, Zhang ZS, et al. (2010) Karyotype analysis in the black bream (hephaestus fuliginosus). Freshwater Fish 40(3): 76-79.

27. Wang SF (2007) Comparative karyotype characterization and chromosomal evolution of six groupers. Xiamen University, Doctoral thesis.

28. Liu SJ (2010) Distant hybridization leads to different ploidy fishes. Sci China Life Sci 53(4): 416-425.
Your next submission with Juniper Publishers will reach you the below assets

- Quality Editorial service

- Swift Peer Review

- Reprints availability

- E-prints Service

- Manuscript Podcast for convenient understanding

- Global attainment for your research

- Manuscript accessibility in different formats ( Pdf, E-pub, Full Text, Audio)

- Unceasing customer service

Track the below URL for one-step submission https://juniperpublishers.com/online-submission.php 